\title{
ODD SINGULAR VECTOR FORMULA FOR GENERAL LINEAR LIE SUPERALGEBRAS
}

\author{
JIE LIU ${ }^{1, a}$, LI LUO ${ }^{2, b}$ AND WEIQIANG WANG ${ }^{2,3, c}$
}

\footnotetext{
${ }^{1}$ Department of mathematics, Shanghai Key Laboratory of Pure Mathematics and Mathematical Practice, East China Normal University, Shanghai 200241, China.

${ }^{a}$ E-mail: jie_liu@math.uni-kiel.de

${ }^{2}$ Department of mathematics, Shanghai Key Laboratory of Pure Mathematics and Mathematical Practice, East China Normal University, Shanghai 200241, China.

${ }^{b}$ E-mail: lluo@math.ecnu.edu.cn

${ }^{3}$ Department of Mathematics, University of Virginia, Charlottesville, VA 22904, USA.

${ }^{c}$ E-mail: ww9c@virginia.edu

\|\|
}

\section{Abstract}

We establish a closed formula for a singular vector of weight $\lambda-\beta$ in the Verma module of highest weight $\lambda$ for Lie superalgebra $\mathfrak{g l}(m \mid n)$ when $\lambda$ is atypical with respect to an odd positive root $\beta$. It is further shown that this vector is unique up to a scalar multiple, and it descends to a singular vector, again unique up to a scalar multiple, in the corresponding Kac module when both $\lambda$ and $\lambda-\beta$ are dominant integral.

\section{Introduction}

For a general basic Lie superalgebra $\mathfrak{g}$ (which is a suitable super generalization of semisimple Lie algebras) with a non-degenerate invariant bilinear form $(\cdot, \cdot)$, the linkage principle is not completely controlled by the Weyl group; cf. the book [1] . Besides the more familiar linkage for weights in a same Weyl group orbit, a so-called $\beta$-atypical weight $\lambda$ can be linked to $\lambda-\beta$ for a positive odd isotropic root $\beta$; here by $\beta$-atypical we mean $(\lambda+\rho, \beta)=0$. This is one of the fundamental differences between representation theories of Lie superalgebras and Lie algebras.

Now let $\mathfrak{g}$ be the general linear Lie superalgebra $\mathfrak{g l}(m \mid n)$ over the complex number field $\mathbb{C}$. The main result of this note is a simple closed formula

Received June 25, 2019.

AMS Subject Classification: 17B10.

Key words and phrases: Lie superalgebra, singular vector. 
for an (odd) singular vector $S_{-\beta} v_{\lambda}^{+}$of weight $\lambda-\beta$ in the Verma module $M(\lambda)$ of highest weight $\lambda$, when $\lambda$ is $\beta$-atypical. We then show that such a singular vector is unique up to a scalar multiple; see Theorem 1. In other words, we have

$$
\operatorname{Hom}_{\mathfrak{g}}(M(\lambda-\beta), M(\lambda))=\mathbb{C} .
$$

This can be regarded as a super analogue of a classical theorem of Verma for semisimple Lie algebras. Serganova [6] showed earlier the Hom space in (11) is non-vanishing by some indirect argument. We note that formulae for various singular vectors associated to an even reflection in a Verma module of a basic Lie superalgebra were recently established in [5]; cf. also [2].

We readily convert our odd singular vector formula to a simple closed formula for the corresponding odd Shapovalov element; see Corollary 3, Musson has studied systematically Shapovalov elements in the setting of basic Lie superalgebras, and in particular, he obtained in [4, Theorems 9.1, 9.2] very different and more involved expressions for odd Shapovalov elements in terms of non-commutative determinants.

For a dominant integral weight $\lambda$ for $\mathfrak{g l}(m \mid n)$, the Kac module $K(\lambda)$ is by definition a maximal finite-dimensional quotient of the Verma module $M(\lambda)$. Assume $\lambda$ is $\beta$-atypical and in addition $\lambda-\beta$ is dominant integral. We show that the aforementioned vector $S_{-\beta} v_{\lambda}^{+}$descends to a (nonzero) singular vector in $K(\lambda)$, and that such a singular vector in $K(\lambda)$ is unique up to a scalar multiple; see Theorem 6. Indeed this uniqueness is equivalent to an earlier simple observation by Serganova that $\operatorname{dim} \operatorname{Hom}_{\mathfrak{g}}(K(\lambda-\beta), K(\lambda)) \leq 1$. Hence we have provided a new constructive proof that $\operatorname{Hom}_{\mathfrak{g}}(K(\lambda-\beta), K(\lambda))=\mathbb{C}$, an old result of Serganova [6, Theorem 5.5].

The identity (11) was also known in 2] for the exceptional Lie superalgebra $D(2,1 ; \zeta)$ where explicit odd singular vector formulas were established. We conjecture that the identity (1) holds for basic Lie superalgebas in general. We show that essentially the same odd singular vector formula for $\mathfrak{g l}(m \mid n)$ is valid for half of the odd isotropic roots of $\mathfrak{o s p}$ Lie superalgebras. It seems highly nontrivial and very interesting to generalize the odd singular vector formula of this paper to the other half of odd roots for osp and the other basic Lie superalgebras. 
Acknowledgement. The authors thank Bin Shu for raising the question about odd singular vectors. LL is partially supported by the Science and Technology Commission of Shanghai Municipality (grant No. 18dz2271000) and the NSF of China (grant No. 11671108, 11871214). WW is partially supported by the NSF grant DMS-1702254, and he thanks ECNU for the hospitality and support during his visit.

\section{Formula for Odd Singular Vectors}

\subsection{The preliminaries}

Let

$$
I=I_{m \mid n}=\{\bar{m}, \overline{m-1}, \ldots, \overline{1}, 1,2, \ldots, n\}
$$

be a set with a total order

$$
\bar{m}<\overline{m-1}<\cdots<\overline{1}<1<2<\cdots<n .
$$

Let $\mathfrak{g}=\mathfrak{g l}(m \mid n)=\mathfrak{g}_{0} \oplus \mathfrak{g}_{\overline{1}}$ be the general linear Lie superalgebra with standard basis $\left\{E_{i, j} \mid i, j \in I\right\}$. Then $\mathfrak{g}$ admits a natural $\mathbb{Z}$-grading $\mathfrak{g}=$ $\mathfrak{g}_{-1} \oplus \mathfrak{g}_{0} \oplus \mathfrak{g}_{1}$ where $\mathfrak{g}_{0}=\mathfrak{g}_{\overline{0}}$ and $\mathfrak{g}_{ \pm 1}$ is of the form

$$
\mathfrak{g}_{-1}=\left\{\left(\begin{array}{cc}
0 & 0 \\
C_{n \times m} & 0
\end{array}\right)\right\}, \quad \mathfrak{g}_{1}=\left\{\left(\begin{array}{cc}
0 & B_{m \times n} \\
0 & 0
\end{array}\right)\right\} .
$$

Let $\mathfrak{h}$ be the Cartan subalgebra consisting of diagonal matrices and let $\mathfrak{g}=$ $\mathfrak{n}^{-} \oplus \mathfrak{h} \oplus \mathfrak{n}^{+}$be a triangular decomposition, where $\mathfrak{n}^{-}$(resp. $\mathfrak{n}^{+}$) consists of lower (resp. upper) triangular matrices. Let $\left\{\delta_{m}, \ldots, \delta_{1}, \epsilon_{1}, \ldots, \epsilon_{n}\right\} \subset \mathfrak{h}^{*}$ be the dual basis of $\left\{E_{\bar{m}, \bar{m}}, \ldots, E_{\overline{1}, \overline{1}}, E_{1,1}, \ldots, E_{n, n}\right\} \subset \mathfrak{h}$. A nondegenerate symmetric form $(\cdot, \cdot)$ on $\mathfrak{h}^{*}$ induced by an invariant symmetric form on $\mathfrak{g}$ is given by

$$
\left(\delta_{i}, \epsilon_{k}\right)=0 \quad \forall i, k, \quad\left(\delta_{i}, \delta_{j}\right)=-\left(\epsilon_{i}, \epsilon_{j}\right)= \begin{cases}1 & \text { if } i=j \\ 0 & \text { if } i \neq j\end{cases}
$$

The set of simple roots and the set of roots are

$$
\Pi=\left\{\delta_{m}-\delta_{m-1}, \ldots, \delta_{2}-\delta_{1}, \delta_{1}-\epsilon_{1}, \epsilon_{1}-\epsilon_{2}, \ldots, \epsilon_{n-1}-\epsilon_{n}\right\},
$$




$$
\Phi=\left\{\delta_{i}-\delta_{j}, \epsilon_{p}-\epsilon_{q}, \pm\left(\delta_{i}-\epsilon_{p}\right) \mid \bar{m} \leq \bar{i} \neq \bar{j} \leq \overline{1}, 1 \leq p \neq q \leq n\right\} .
$$

Furthermore, the sets of positive even roots and positive odd roots are given by

$$
\begin{aligned}
& \Phi_{0}^{+}=\left\{\delta_{i}-\delta_{j}, \epsilon_{p}-\epsilon_{q} \mid \bar{m} \leq \bar{i}<\bar{j} \leq \overline{1}, 1 \leq p<q \leq n\right\}, \\
& \Phi_{1}^{+}=\left\{\delta_{i}-\epsilon_{p} \mid \bar{m} \leq \bar{i}<1 \leq p \leq n\right\} .
\end{aligned}
$$

The set of even roots and the set of odd roots are $\Phi_{0}=\Phi_{0}^{+} \cup\left(-\Phi_{0}^{+}\right)$and $\Phi_{1}=\Phi_{1}^{+} \cup\left(-\Phi_{1}^{+}\right)$, respectively. The root space decomposition is $\mathfrak{g}=\mathfrak{h} \oplus$ $\left(\bigoplus_{\alpha \in \Phi} \mathfrak{g}_{\alpha}\right)$, where

$$
\mathfrak{g}_{\delta_{i}-\delta_{j}}=\mathbb{C} E_{\bar{i}, \bar{j}}, \quad \mathfrak{g}_{\epsilon_{p}-\epsilon_{q}}=\mathbb{C} E_{p, q}, \quad \mathfrak{g}_{\delta_{i}-\epsilon_{p}}=\mathbb{C} E_{\bar{i}, p}, \quad \mathfrak{g}_{\epsilon_{p}-\delta_{i}}=\mathbb{C} E_{p, \bar{i}} .
$$

A weight $\lambda \in \mathfrak{h}^{*}$ is integral if $(\lambda, \alpha) \in \mathbb{Z}$ for all $\alpha \in \Phi_{0}$, and is dominant if $(\lambda, \alpha) \in \mathbb{Z}_{\geq 0}$ for all $\alpha \in \Phi_{0}^{+}$. We denote by $P^{+}$the set of all integral dominant weights. We recall the Weyl vector

$$
\rho=\frac{1}{2}\left(\sum_{\alpha \in \Phi_{0}^{+}} \alpha-\sum_{\alpha \in \Phi_{1}^{+}} \alpha\right)=\sum_{i=1}^{m} i \delta_{i}-\sum_{j=1}^{n} j \epsilon_{j}-\frac{m+n+1}{2} \mathbf{1}_{m \mid n},
$$

where $\mathbf{1}_{m \mid n}=\sum_{i=1}^{m} \delta_{i}-\sum_{j=1}^{n} \epsilon_{j}$. If there is a positive odd root $\beta \in \Phi_{1}^{+}$such that $(\lambda+\rho, \beta)=0$, then the weight $\lambda$ is called atypical (or more precisely, $\beta$-atypical).

In this paper, a weight $\lambda=a_{m} \delta_{m}+\cdots+a_{1} \delta_{1}+b_{1} \epsilon_{1}+\cdots+b_{n} \epsilon_{n} \in \mathfrak{h}^{*}$ will be denoted by

$$
\lambda=\left(a_{m}, \ldots, a_{1} \mid b_{1}, \ldots, b_{n}\right) .
$$

The following clearly holds:

$$
\begin{aligned}
& \lambda \text { is integral } \Leftrightarrow a_{i}-a_{j}, b_{p}-b_{q} \in \mathbb{Z}, \forall \bar{m} \leq \bar{i}<\bar{j} \leq \overline{1}, 1 \leq p<q \leq n ; \\
& \lambda \text { is dominant } \Leftrightarrow a_{i}-a_{j}, b_{p}-b_{q} \geq 0, \forall \bar{m} \leq \bar{i}<\bar{j} \leq \overline{1}, 1 \leq p<q \leq n ;
\end{aligned}
$$

$\lambda$ is $\left(\delta_{s}-\epsilon_{t}\right)$-atypical $\Leftrightarrow a_{s}+b_{t}+s-t=0$. 


\subsection{Singular vectors in Verma modules}

A non-zero vector $v$ in a $\mathfrak{g}$-module $V$ is called singular if $\mathfrak{n}^{+} \cdot v=0$. Recall the Verma module of highest weight $\lambda$ is $M(\lambda)=\operatorname{Ind}_{\mathfrak{h} \oplus \mathfrak{n}^{+}}^{\mathfrak{g}} \mathbb{C} 1_{\lambda}$, where $h \cdot 1_{\lambda}=\lambda(h) 1_{\lambda}$ and $\mathfrak{n}^{+} \cdot 1_{\lambda}=0$; the highest weight vector of $M(\lambda)$ is denoted by $v_{\lambda}^{+}=1 \otimes 1_{\lambda}$.

Let $\beta=\delta_{s}-\epsilon_{t}$. Assume $\lambda=\left(a_{m}, a_{m-1}, \ldots, a_{1} \mid b_{1}, b_{2}, \ldots, b_{n}\right)$ is a $\beta$-atypical weight. Define a sequence of scalars

$$
\begin{gathered}
c_{\overline{s-1}}=a_{s}-a_{s-1}, c \overline{s-2}=a_{s}-a_{s-2}+1, \ldots, c_{\overline{1}}=a_{s}-a_{1}+s-2 ; \\
c_{t-1}=b_{t}-b_{t-1}-1, c_{t-2}=b_{t}-b_{t-2}-2, \ldots, c_{1}=b_{t}-b_{1}-t+1 .
\end{gathered}
$$

Denote

$\mathcal{A}=\left\{J=\left\{j_{1}, j_{2}, \ldots, j_{p}\right\} \subset I \mid \bar{s}<j_{1}<j_{2}<\cdots<j_{p}<t, 0 \leq p \leq s+t-2\right\}$,

where it is understood that $J=\emptyset$ if $p=0$. Then

$$
\left\{E_{J}:=E_{t, j_{p}} E_{j_{p}, j_{p-1}} \cdots E_{j_{1}, \bar{s}} \mid J=\left\{j_{1}, j_{2}, \ldots, j_{p}\right\} \in \mathcal{A}\right\}
$$

forms a basis of the $(-\beta)$-weight subspace of $\mathrm{U}\left(\mathfrak{n}^{-}\right)$; here it is understood that $E_{\emptyset}=E_{t, \bar{s}}$. For any $J \in \mathcal{A}$, define

$$
d_{J}=\prod_{\bar{s}<k<t, k \notin J} c_{k}
$$

where it is understood that $d_{\{\overline{s-1}, \overline{s-2}, \ldots, \overline{1}, 1,2, \ldots, t-1\}}=1$. Introduce the following element in $U\left(\mathfrak{n}^{-}\right)$:

$$
S_{-\beta}=\sum_{J \in \mathcal{A}} d_{J} E_{J}
$$

which contains a unique term (called the leading term) with $J$ of maximal cardinality $E_{t, t-1} \cdots E_{2,1} E_{1, \overline{1}} E_{\overline{1}, \overline{2}} \cdots E_{\overline{s-1}, \bar{s}}$ of coefficient 1 .

Theorem 1. Let $\beta=\delta_{s}-\epsilon_{t}$ and let $\lambda=\left(a_{m}, a_{m-1}, \ldots, a_{1} \mid b_{1}, b_{2}, \ldots, b_{n}\right)$ be a $\beta$-atypical weight. Then the element $S_{-\beta} v_{\lambda}^{+}$is the unique (up to a nonzero scalar multiple) singular vector in $M(\lambda)$ of weight $\lambda-\beta$. In particular, we have

$$
\operatorname{Hom}_{\mathfrak{g}}(M(\lambda-\beta), M(\lambda))=\mathbb{C}
$$


Proof. To show that $S_{-\beta} v_{\lambda}^{+}$is singular, it suffices to verify that $E_{\bar{i}, \overline{i-1}} \cdot S_{-\beta} v_{\lambda}^{+}=E_{j-1, j} \cdot S_{-\beta} v_{\lambda}^{+}=E_{\overline{1}, 1} \cdot S_{-\beta} v_{\lambda}^{+}=0, \quad(\forall 1<i \leq m, 1<j \leq n)$.

Indeed, it is trivial that $E_{j-1, j} \cdot S_{-\beta} v_{\lambda}^{+}=0 \quad(\forall j>t)$. Furthermore,

$$
\begin{aligned}
E_{t-1, t} \cdot S_{-\beta} v_{\lambda}^{+} & =E_{t-1, t} \cdot \sum_{t-1 \in J \in \mathcal{A}}\left(d_{J}\left(E_{t, t-1} E_{t-1, \sharp} \cdots\right)+d_{J} c_{t-1}\left(E_{t, \sharp} \cdots\right)\right) \\
& =\sum_{t-1 \in J \in \mathcal{A}} d_{J}\left(b_{t-1}-b_{t}+1+c_{t-1}\right)\left(E_{t-1, \sharp} \cdots\right)=0,
\end{aligned}
$$

where the last equation uses the identity $b_{t-1}-b_{t}+1+c_{t-1}=0$ which follows by (4).

For $1<j<t$, we have

$$
\begin{aligned}
& E_{j-1, j} \cdot S_{-\beta} v_{\lambda}^{+} \\
& =E_{j-1, j} \cdot \sum_{j, j-1 \in J \in \mathcal{A}}\left(\begin{array}{l}
d_{J}\left(\cdots E_{*, j} E_{j, j-1} E_{j-1, \sharp} \cdots\right)+d_{J} c_{j}\left(\cdots E_{*, j-1} E_{j-1, \sharp} \cdots\right) \\
+d_{J} c_{j-1}\left(\cdots E_{*, j} E_{j, \sharp} \cdots\right)+d_{J} c_{j} c_{j-1}\left(\cdots E_{*, \sharp} \cdots\right)
\end{array}\right) v_{\lambda}^{+} \\
& =\sum_{j, j-1 \in J \in \mathcal{A}} d_{J}\left(b_{j-1}-b_{j}+1-c_{j}+c_{j-1}\right)\left(\cdots E_{*, j} E_{j-1, \sharp} \cdots\right) v_{\lambda}^{+}=0,
\end{aligned}
$$

where the last equation uses the identity $b_{j-1}-b_{j}+1-c_{j}+c_{j-1}=0$ which follows by (44).

Summarizing we have known $E_{j-1, j} \cdot S_{-\beta} v_{\lambda}^{+}=0(\forall 1<j \leq n)$. In an entirely similar way, we can show $E_{\bar{i}, \bar{i}-1} \cdot S_{-\beta} v_{\lambda}^{+}=0(\forall 1<i \leq m)$.

In addition, we compute that

$$
\begin{aligned}
& E_{\overline{1}, 1} \cdot S_{-\beta} v_{\lambda}^{+} \\
& =E_{\overline{1}, 1} \cdot \sum_{\overline{1}, 1 \in J \in \mathcal{A}}\left(\begin{array}{l}
d_{J}\left(\cdots E_{*, 1} E_{1, \overline{1}} E_{\overline{1}, \sharp} \cdots\right)+d_{J} c_{\overline{1}}\left(\cdots E_{*, 1} E_{1, \sharp} \cdots\right) \\
+d_{J} c_{1}\left(\cdots E_{*, \overline{1}} E_{\overline{1}, \sharp} \cdots\right)+d_{J} c_{\overline{1}} c_{1}\left(\cdots E_{*, \sharp} \cdots\right)
\end{array}\right) v_{\lambda}^{+} \\
& =\sum_{\overline{1}, 1 \in J \in \mathcal{A}} d_{J}\left(b_{1}+a_{1}+1+c_{1}+c_{\overline{1}}\right)\left(\cdots E_{*, 1} E_{\overline{1}, \sharp} \cdots\right) v_{\lambda}^{+} \\
& =\sum_{\overline{1}, 1 \in J \in \mathcal{A}} d_{J}\left(a_{s}+b_{t}+s-t\right)\left(\cdots E_{*, 1} E_{\overline{1}, \sharp} \cdots\right) v_{\lambda}^{+} \quad \text { by (3) (4) (4) } \\
& =0 \quad \text { by (2). }
\end{aligned}
$$


(In the above calculation, we implicitly assume $s, t>1$. If either $s=1$ or $t=1$, the argument is similar and much easier.)

Hence we have verified that $S_{-\beta} v_{\lambda}^{+}$is a singular vector of weight $(\lambda-\beta)$ in $M(\lambda)$.

It remains to prove the uniqueness. In a nutsell, the reason for the uniqueness is that the coefficients of summands in $S_{-\beta} v_{\lambda}^{+}$(see (7)) are determined recursively by the requirement that $S_{-\beta} v_{\lambda}^{+}$is singular once the leading coefficient is fixed. More precisely, let

$$
S_{-\beta}^{\prime}=\sum_{J \in \mathcal{A}} z_{J} E_{t, j_{p}} E_{j_{p}, j_{p-1}} \cdots E_{j_{1}, \bar{s}}, \quad\left(\text { for } z_{J} \in \mathbb{C}\right)
$$

be a vector of weight $\lambda-\beta$ which is annihilated by $\mathfrak{n}^{+}$. Subtracting $S_{-\beta}^{\prime}$ by a suitable multiple of $S_{-\beta}$ if necessary, we may assume

$$
z_{\{\overline{s-1}, \overline{s-2}, \ldots, \overline{1}, 1,2, \ldots, t-1\}}=0 .
$$

We will show that $z_{J}=0$ for any $J \in \mathcal{A}$ by downward induction on the cardinality $|J|$.

The base step is (8), as $J=\{\overline{s-1}, \ldots, \overline{1}, 1, \ldots, t-1\}$ has the maximal cardinality among $J \in \mathcal{A}$ in (5). Suppose we have known that $z_{J^{\prime}}=0$ for any $J^{\prime} \in \mathcal{A}$ with $\left|J^{\prime}\right|>k$. We consider $J \in \mathcal{A}$ with $|J|=k$.

If $t-1 \notin J$, it follows from $E_{t-1, t} \cdot S_{-\beta}^{\prime} v_{\lambda}^{+}=0$ that $z_{J \cup\{t-1\}}\left(b_{t-1}-\right.$ $\left.b_{t}+1\right)+z_{J}=0$. Thus $z_{J}=0$ because of $z_{J \cup\{t-1\}}=0$ by the inductive assumption.

If $t-1 \in J$ but $t-2 \notin J$, it follows from $E_{t-2, t-1} \cdot S_{-\beta}^{\prime} v_{\lambda}^{+}=0$ that

$$
z_{J \cup\{t-2\}}\left(b_{t-2}-b_{t-1}+1\right)+z_{J}-z_{(J \cup\{t-2\}) \backslash\{t-1\}}=0 .
$$

Thanks to $z_{J \cup\{t-2\}}=0$ by inductive assumption and $z_{(J \cup\{t-2\}) \backslash\{t-1\}}=0$ established in the previous case, we obtain $z_{J}=0$.

Similarly, we can show $z_{J}=0$ if $t-1, t-2 \in J$ but $t-3 \notin J$, by $E_{t-3, t-2} \cdot S_{-\beta}^{\prime} v_{\lambda}^{+}=0$. Repeating this procedure, we see that $z_{J}=0$ for all $J \in \mathcal{A}$ with $|J|=k$. 
Therefore we obtain that $S_{-\beta}^{\prime}=0$, and the uniqueness follows. The uniqueness of the singular vector implies that (and is equivalent to) $\operatorname{Hom}_{\mathfrak{g}}(M(\lambda-\beta), M(\lambda))=\mathbb{C}$.

It was known [6, Lemma 5.4] that $\operatorname{Hom}_{\mathfrak{g}}(M(\lambda-\beta), M(\lambda)) \neq 0$.

Example 2. Let us write down explicitly some cases of odd singular vectors in Theorem 11 Keep the notation $\lambda=\left(a_{m}, \ldots, a_{1} \mid b_{1}, \ldots, b_{n}\right) \in \mathfrak{h}^{*}$.

(1) Let $\beta=\delta_{2}-\epsilon_{1}$ and let $\lambda$ be $\beta$-atypical, i.e., $a_{2}+b_{1}+1=0$. Then

$$
S_{-\beta}=E_{1, \overline{1}} E_{\overline{1}, \overline{2}}+\left(a_{2}-a_{1}\right) E_{1, \overline{2}} .
$$

(2) Let $\beta=\delta_{3}-\varepsilon_{1}$ and let $\lambda$ be $\beta$-atypical, i.e., $a_{3}+b_{1}+2=0$. Then

$$
\begin{aligned}
S_{-\beta}= & E_{1, \overline{1}} E_{\overline{1}, \overline{2}} E_{\overline{2}, \overline{3}}+\left(a_{3}-a_{2}\right) E_{1, \overline{1}} E_{\overline{1}, \overline{3}} \\
& +\left(a_{3}-a_{1}+1\right) E_{1, \overline{2}} E_{\overline{2}, \overline{3}}+\left(a_{3}-a_{1}+1\right)\left(a_{3}-a_{2}\right) E_{1, \overline{3}} .
\end{aligned}
$$

(3) Let $\beta=\delta_{2}-\varepsilon_{2}$ and let $\lambda$ be $\beta$-atypical, i.e., $a_{2}+b_{2}=0$. Then

$$
\begin{aligned}
S_{-\beta}= & E_{2,1} E_{1, \overline{1}} E_{\overline{1}, \overline{2}}+\left(a_{2}-a_{1}\right) E_{2,1} E_{1, \overline{2}} \\
& +\left(b_{2}-b_{1}-1\right) E_{2, \overline{1}} E_{\overline{1}, \overline{2}}+\left(a_{2}-a_{1}\right)\left(b_{2}-b_{1}-1\right) E_{2, \overline{2}} .
\end{aligned}
$$

Inspired by the formulas (3)-(44), we introduce the following elements in $\mathbb{C}+\mathfrak{h} \subset U(\mathfrak{h}):$

$$
\begin{aligned}
C_{\overline{s-1}} & =E_{\bar{s}, \bar{s}}-E_{\overline{s-1}, \overline{s-1}}, C \overline{s-2}=E_{\bar{s}, \bar{s}}-E_{\overline{s-2}, \overline{s-2}}+1, \cdots, \\
C_{\overline{1}} & =E_{\bar{s}, \bar{s}}-E_{\overline{1}, \overline{1}}+s-2, \\
C_{t-1} & =E_{t, t}-E_{t-1, t-1}-1, \quad C_{t-2}=E_{t, t}-E_{t-2, t-2}-2, \ldots, \\
C_{1} & =E_{t, t}-E_{1,1}-t+1 .
\end{aligned}
$$

Denote the Borel subalgebra $\mathfrak{b}^{-}=\mathfrak{n}^{-} \oplus \mathfrak{h}$. By definition, a Shapovalov element is an element $\theta_{\beta} \in \mathrm{U}\left(\mathfrak{b}^{-}\right)_{-\beta}$ such that $\theta_{\beta} v_{\lambda}^{+}$is a singular vector of weight $\lambda-\beta$ for any $\beta$-atypical weight $\lambda$. We have the following corollary to Theorem 1 .

Corollary 3. Let $\beta=\delta_{s}-\epsilon_{t} \in \Phi_{1}^{+}$. Then the element

$$
\theta_{\beta}=\sum_{J \in \mathcal{A}} E_{J}\left(\prod_{\bar{s}<k<t, k \notin J} C_{k}\right)
$$


is the unique element in $\mathrm{U}\left(\mathfrak{b}^{-}\right)_{-\beta}$ which satisfies $\theta_{\beta} v_{\lambda}^{+}=S_{-\beta} v_{\lambda}^{+}$for any $\beta$-atypical weight $\lambda$; hence it is a Shapovalov element.

Some other more complicated expressions for a Shapovalov element associated to $\beta \in \Phi_{1}^{+}$in terms of non-commutative determinants were also obtained by Musson in [4, Theorems 9.1, 9.2]. By the uniqueness, these two versions of odd Shapovalov elements coincide.

Remark 4. It was brought to our attention by an anonymous expert that $S_{-\beta}$ above are examples of lowering operators (in different forms) which have been studied in the literature; see [3, §4] (and in particular Theorem 4.11 therein) where general lowering operators were constructed and studied for general linear Lie superalgebra $\mathfrak{g}$.

\subsection{Singular vectors in Kac modules}

For any dominant integral weight $\lambda \in P^{+}$, let $L^{0}(\lambda)$ be the finite dimensional irreducible $\mathfrak{g}_{0}$-module of highest weight $\lambda$. Extending the $\mathfrak{g}_{0^{-}}$ module $L^{0}(\lambda)$ to a $\mathfrak{g}_{0} \oplus \mathfrak{g}_{1}$-module by a trivial $\mathfrak{g}_{1}$-action, we define the (finite-dimensional) Kac module as

$$
K(\lambda):=\operatorname{Ind}_{\mathfrak{g}_{0} \oplus \mathfrak{g}_{1}}^{\mathfrak{g}} L^{0}(\lambda) .
$$

For any dominant integral weight $\lambda=\left(a_{m}, a_{m-1}, \ldots, a_{1} \mid b_{1}, b_{2}, \ldots, b_{n}\right)$ $\in P^{+}$, it is well known that

$$
K(\lambda)=M(\lambda) / I_{\lambda}
$$

where $I_{\lambda}$ is the $\mathfrak{g}$-submodule generated by the singular vectors

$$
\left\{E_{\overline{i-1}, i}^{a_{i}-a_{i-1}+1} v_{\lambda}^{+}, E_{j+1, j}^{b_{j}-b_{j+1}+1} v_{\lambda}^{+} \mid 1<i \leq m, 1 \leq j<n\right\} .
$$

Let $J_{\lambda}$ be the $\mathfrak{n}^{-}$-submodule of $M(\lambda)$ generated by

$$
\left\{E_{\overline{i-1}, i} v_{\lambda}^{+}, E_{j+1, j} v_{\lambda}^{+} \mid 1<i \leq m, 1 \leq j<n\right\} .
$$




\section{Lemma 5.}

(1) For $n \geq i>j \geq 1$ or $\overline{1} \geq i>j \geq \bar{m}$, we have $E_{i, j} v_{\lambda}^{+} \in J_{\lambda}$.

(2) For $n \geq t \geq 1>\bar{s} \geq \bar{m}$, we have $E_{t, \bar{s}} v_{\lambda}^{+} \notin J_{\lambda}$.

Proof. (1) For any $n \geq i>j \geq 1$, we have

$$
\begin{aligned}
E_{i, j} v_{\lambda}^{+} & =E_{i, j+1} E_{j+1, j} v_{\lambda}^{+}-E_{j+1, j} E_{i, j+1} v_{\lambda}^{+} \\
& \equiv-E_{j+1, j} E_{i, j+1} v_{\lambda}^{+} \equiv \cdots \\
& \equiv(-1)^{i-j-1} E_{j+1, j} E_{j+2, j+1} \cdots E_{i, i-1} v_{\lambda}^{+} \equiv 0 \quad\left(\bmod J_{\lambda}\right) .
\end{aligned}
$$

The remaining case (with $\overline{1} \geq i>j \geq \bar{m}$ ) follows by an entirely similar argument.

(2) Let $\mathfrak{n}_{\overline{0}}^{-}=\mathfrak{n}^{-} \cap \mathfrak{g}_{\overline{0}}, \mathfrak{n}_{\overline{1}}^{+}=\mathfrak{n}^{+} \cap \mathfrak{g}_{\overline{1}}$, and so that $\mathfrak{n}^{-}=\mathfrak{n}_{\overline{0}}^{-} \oplus \mathfrak{n}_{\overline{1}}^{-}$. Thanks to the PBW theorem, we have $\mathrm{U}\left(\mathfrak{n}^{-}\right)=\mathrm{U}\left(\mathfrak{n}_{\overline{1}}^{-}\right) \mathrm{U}\left(\mathfrak{n}_{\overline{0}}^{-}\right)=\mathrm{U}\left(\mathfrak{n}_{\overline{1}}^{-}\right) \oplus \mathrm{U}\left(\mathfrak{n}^{-}\right) \mathfrak{n}_{\overline{0}}^{-}$. We have $E_{t, \bar{s}} \in \mathrm{U}\left(\mathfrak{n}_{1}^{-}\right)$, and hence $E_{t, \bar{s}} \notin \mathrm{U}\left(\mathfrak{n}^{-}\right) \mathfrak{n}_{\overline{0}}^{-}$. Therefore we have $E_{t, \bar{s}} v_{\lambda}^{+} \notin \mathrm{U}\left(\mathfrak{n}^{-}\right) \mathfrak{n}_{\overline{0}}^{-} v_{\lambda}^{+}=J_{\lambda}$.

We continue to denote by $v_{\lambda}^{+}$the highest weight vector in $K(\lambda)$.

Theorem 6. Let $\beta \in \Phi_{1}^{+}, \lambda \in P^{+}$and $\lambda-\beta \in P^{+}$be such that $(\lambda+\rho, \beta)=0$. Then $S_{-\beta} v_{\lambda}^{+}$is a singular vector of weight $\lambda-\beta$, unique up to a scalar multiple, in the Kac module $K(\lambda)$. In particular, we have

$$
\operatorname{Hom}_{\mathfrak{g}}(K(\lambda-\beta), K(\lambda))=\mathbb{C} \text {. }
$$

Proof. Recall $K(\lambda)=M(\lambda) / I_{\lambda}$ in (10). We check that $S_{-\beta} v_{\lambda}^{+}$(regarded as a vector in $M(\lambda)$ ) does not lie in the submodule $I_{\lambda}$. To that end, let

$$
\mathcal{B}=\left\{J=\left\{j_{1}, j_{2}, \ldots, j_{p}\right\} \subset I \mid 1 \leq j_{1}<j_{2}<\cdots<j_{p}<t, 0 \leq p \leq t-1\right\} \subset \mathcal{A},
$$

where it is understood that $J=\emptyset$ if $p=0$. Moreover, below it is understood that $E_{t, j_{p}} E_{j_{p}-j_{p-1}} \cdots E_{j_{1}, \bar{s}}=E_{t, \bar{s}}$ if $p=0$. By applying Lemma 5 (1) repeatedly we have

$$
\begin{aligned}
S_{-\beta} v_{\lambda}^{+} & \equiv \sum_{J \in \mathcal{B}} d_{J} E_{t, j_{p}} E_{j_{p}-j_{p-1}} \cdots E_{j_{1}, \bar{s}} v_{\lambda}^{+} \\
& \equiv\left(\sum_{J \in \mathcal{B}} d_{J}\right) E_{t, \bar{s}} v_{\lambda}^{+}
\end{aligned}
$$




$$
=\left(\prod_{\bar{s}<i \leq \overline{1}} c_{i}\right)\left(\prod_{1 \leq j<t}\left(1+c_{j}\right)\right) E_{t, \bar{s}} v_{\lambda}^{+} \quad\left(\bmod J_{\lambda}\right) .
$$

We know $E_{t, \bar{s}} v_{\lambda}^{+} \notin J_{\lambda}$ by Lemma 5 (2). Note that $\lambda-\beta \in P^{+}$implies $c_{i}>0$ for $\bar{s}<i \leq \overline{1}$ and $1+c_{j}<0$ for $1 \leq j<t$. It follows by (14) that $S_{-\beta} v_{\lambda}^{+} \notin J_{\lambda}$.

Since the $\mathfrak{n}^{-}$-submodule generated by the singular vectors in (11) is equal to $I_{\lambda}$, we have $I_{\lambda} \subseteq J_{\lambda}$ by the definition (12) of $J_{\lambda}$, and hence $S_{-\beta} v_{\lambda}^{+} \notin I_{\lambda}$. We conclude by Theorem 1 that $S_{-\beta} v_{\lambda}^{+}$descends to a singular vector in the Kac module $K(\lambda)$.

To complete the proof of the theorem, it remains to prove that

$$
\operatorname{dim} \operatorname{Hom}_{\mathfrak{g}}(K(\lambda-\beta), K(\lambda)) \leq 1,
$$

or equivalently, by Frobenius reciprocity,

$$
\operatorname{dim} \operatorname{Hom}_{\mathfrak{g}_{\overline{0}}}\left(L^{0}(\lambda-\beta), \Lambda\left(\mathfrak{g}_{-1}\right) \otimes L^{0}(\lambda)\right) \leq 1 .
$$

The statement (15) was stated by Serganova in [6, Proof of Theorem 5.5] who skipped the proof. Let us provide a short proof below (which is a simplified version of our original argument thanks to inputs from Shun-Jen Cheng). A singular vector for the $\mathfrak{g}_{0}$-module $\Lambda\left(\mathfrak{g}_{-1}\right) \otimes L^{0}(\lambda)$ must be of the form $x \otimes v_{\lambda}^{+}+\cdots \otimes U\left(\mathfrak{n}_{\overline{0}}^{-}\right) \mathfrak{n}_{\overline{0}}^{-} v_{\lambda}^{+}$for some $0 \neq x \in \Lambda\left(\mathfrak{g}_{-1}\right)$, and so is of weight $\lambda+\mu$ for some weight $\mu$ for $\Lambda\left(\mathfrak{g}_{-1}\right)$. Now observe that the $(-\beta)$-weight subspace $\Lambda\left(\mathfrak{g}_{-1}\right)_{-\beta}=\Lambda^{1}\left(\mathfrak{g}_{-1}\right)_{-\beta}$ is one-dimensional; hence (15) follows.

The proof of the theorem is completed.

Remark 7. The equality (13) was proved by Serganova in $[6$, Theorem $5.5]$ in a different approach. Our proof that $\operatorname{Hom}_{\mathfrak{g}}(K(\lambda-\beta), K(\lambda)) \neq 0$ is constructive and direct.

Remark 8. The assumption $\lambda-\beta \in P^{+}$in Theorem [6 cannot be removed. Consider $\mathfrak{g}=\mathfrak{g l}(2 \mid 1)$ and $\beta=\delta_{2}-\varepsilon_{1} \in \Phi_{1}^{+}$. A weight $\lambda=\left(a_{2}, a_{1} \mid b_{1}\right)$ with $b_{1}=-a_{2}-1$ is $\beta$-atypical. The formula (7) reads $S_{-\beta}=E_{1, \overline{1}} E_{\overline{1}, \overline{2}}+c_{\overline{1}} E_{1, \overline{2}}$. If $a_{1}=a_{2}$, then by (3) $c_{\overline{1}}=0$ and hence $S_{-\beta} v_{\lambda}^{+}=0$ in $K(\lambda)$. 


\subsection{Other basic types}

Verma $\mathfrak{g}$-modules $M(\lambda)$ can be defined as usual with respect to a triangular decomposition $\mathfrak{g}=\mathfrak{n}^{-} \oplus \mathfrak{h} \oplus \mathfrak{n}^{+}$for any basic Lie superalgebra $\mathfrak{g}$ (cf. [1, Chapter 1] for basic Lie superalgebras with standard choices of positive root systems).

Conjecture 9. Let $\mathfrak{g}$ be a basic Lie superalgebra. Assume a weight $\lambda$ is $\beta$-atypical for an isotropic positive odd root $\beta$. Then

$$
\operatorname{Hom}_{\mathfrak{g}}(M(\lambda-\beta), M(\lambda))=\mathbb{C}
$$

We note that via the study of Shapovalov elements Musson [4] has already established the Hom space in (1) is non-vanishing. The conjecture holds for $\mathfrak{g}=\mathfrak{g l}(m \mid n)$ by Theorem 11. It also holds for the exceptional Lie superalgebra $D(2 \mid 1 ; \zeta)$ by [2, Lemma 2.3], where formulas for the singular vectors are given.

Below we focus on the ortho-symplectic Lie superalgebras osp. A standard set of simple roots for $\mathfrak{g}=\mathfrak{o s p}(2 m \mid 2 n)$ is

$$
\Pi=\left\{\delta_{i+1}-\delta_{i}, \delta_{1}-\epsilon_{1}, \epsilon_{j}-\epsilon_{j+1}, \epsilon_{m-1}+\epsilon_{m} \mid 1 \leq i<n, 1 \leq j<m\right\},
$$

and the sets of positive roots and roots are

$$
\begin{aligned}
\Phi^{+} & =\left\{\delta_{i} \pm \delta_{j}, \delta_{i} \pm \epsilon_{k}, \epsilon_{k} \pm \epsilon_{l}, 2 \delta_{i} \mid i>j, k<l\right\} \\
\Phi & =\Phi^{+} \cup\left(-\Phi^{+}\right) .
\end{aligned}
$$

We have $\rho=(n-m, \ldots, 2-m, 1-m \mid m-1, m-2, \ldots, 0)$. Moreover, the set of positive odd roots is

$$
\Phi_{1}^{+}=\left\{\delta_{i} \pm \epsilon_{k} \mid 1 \leq i \leq n, 1 \leq k \leq m\right\} .
$$

For $\beta=\delta_{s} \pm \epsilon_{t} \in \Phi_{1}^{+}$, a weight $\lambda=\sum_{i=1}^{n} a_{i} \delta_{i}+\sum_{k=1}^{m} b_{k} \epsilon_{k}=\left(a_{n}, \ldots, a_{1} \mid b_{1}\right.$, $\left.\ldots, b_{m}\right)$ is $\beta$-atypical (that is, $(\lambda+\rho, \beta)=0$ ) if and only if $a_{s}-m+s=$ $\pm\left(b_{t}+m-t\right)$.

Denote by $e_{\alpha}(\alpha \in \Phi)$ the Chevalley generators of $\mathfrak{g}$. Regarding $\mathfrak{g}$ as a subalgebra of $\mathfrak{g l}(2 m \mid 2 n)$, the generators can be chosen explicitly as follows 
(cf. [1]):

$$
\begin{aligned}
e_{2 \delta_{i}} & =E_{\bar{i}, \overline{i+n}}, & e_{-2 \delta_{i}} & =E_{\overline{i+n}, \bar{i}}, \\
e_{\delta_{i}+\delta_{j}} & =E_{\bar{i}, \overline{j+n}}+E_{\bar{j}, \overline{i+n}}, & e_{-\delta_{i}-\delta_{j}} & =E_{\overline{j+n}, \bar{i}}+E_{\overline{i+n}, \bar{j}}, \quad(i \neq j) \\
e_{\delta_{i}-\delta_{j}} & =E_{\bar{i}, \bar{j}}-E_{\overline{j+n}, \overline{i+n}}, & e_{\epsilon_{k}-\epsilon_{l}} & =E_{k l}-E_{l+m, k+m}, \\
e_{\epsilon_{k}+\epsilon_{l}} & =E_{k, l+m}-E_{l, k+m}, & e_{-\epsilon_{k}-\epsilon_{l}} & =E_{l+m, k}-E_{k+m, l}, \quad(k<l) \\
e_{\delta_{i}+\epsilon_{k}} & =E_{k, \overline{i+n}}+E_{\bar{i}, k+m}, & e_{-\delta_{i}-\epsilon_{k}} & =E_{k+m, \bar{i}}-E_{\overline{i+n}, k}, \\
e_{\delta_{i}-\epsilon_{k}} & =E_{k+m, \overline{i+n}}+E_{\bar{i}, k}, & e_{-\delta_{i}+\epsilon_{k}} & =E_{k, \bar{i}}-E_{\overline{i+n}, k+m} .
\end{aligned}
$$

(The generators for $\mathfrak{o s p}(2 m+1 \mid 2 n)$ can be similarly chosen; cf. [1].)

Recall the set $\mathcal{A}$ in (5). For any $J=\left\{j_{1}, j_{2}, \ldots, j_{p}\right\} \in \mathcal{A}$, we set

$$
e_{J}=e_{\epsilon_{t}-\epsilon_{j_{p}}} e_{\epsilon_{j_{p}}-\epsilon_{j_{p-1}}} \cdots e_{\epsilon_{j_{1}}-\epsilon_{\bar{s}}}
$$

where we use the convention $\epsilon_{\bar{i}}:=\delta_{i}$. It is understood $e_{\emptyset}=e_{\epsilon_{t}-\delta_{s}}$. Recall the scalar $d_{J}$ in (6). Now we set $S_{-\beta}=\sum_{J \in \mathcal{A}} d_{J} e_{J}$.

Proposition 10. Let $\mathfrak{g}=\mathfrak{o s p}(2 m \mid 2 n)$ or osp $(2 m+1 \mid 2 n)$. Let $\beta=\delta_{s}-\epsilon_{t}$. If a weight $\lambda=\left(a_{n}, \ldots, a_{1} \mid b_{1}, \ldots, b_{m}\right)$ is $\beta$-atypical, then the element $S_{-\beta} v_{\lambda}^{+}$ is the unique (up to a nonzero scalar multiple) singular vector in $M(\lambda)$ of weight $\lambda-\beta$.

Proof. Follows by the same proof as for Theorem 1 .

In particular, Conjecture 9 holds for odd roots of the form $\beta=\delta_{s}-\epsilon_{t}$ of the osp Lie superalgebras. This singular vector formula does not easily generalize to the setting of an odd root of $\mathfrak{o s p}$ of the form $\delta_{s}+\epsilon_{t}$. We end with an example of $\mathfrak{o s p}$ of low rank supporting the conjecture in this case.

Example 11. Let $\mathfrak{g}=\mathfrak{o s p}(6 \mid 2), \rho=(-2 \mid 2,1,0)$ and $\beta=\delta_{1}+\epsilon_{1}$. Assume $\lambda=\left(a \mid b_{1}, b_{2}, b_{3}\right)$ with $a=b_{1}+4$. Then $(\lambda+\rho, \beta)=0$. Define

$$
\begin{aligned}
S_{-\beta}= & e_{\epsilon_{3}-\epsilon_{2}} e_{-\epsilon_{3}-\epsilon_{2}} e_{\epsilon_{2}-\epsilon_{1}}^{2} e_{\epsilon_{1}-\delta}-\left(b_{2}+b_{3}+2\right) e_{\epsilon_{3}-\epsilon_{2}} e_{-\epsilon_{3}-\epsilon_{1}} e_{\epsilon_{2}-\epsilon_{1}} e_{\epsilon_{1}-\delta} \\
& -\left(b_{2}-b_{3}+2\right) e_{-\epsilon_{3}-\epsilon_{2}} e_{\epsilon_{3}-\epsilon_{1}} e_{\epsilon_{2}-\epsilon_{1}} e_{\epsilon_{1}-\delta}-\left(2 b_{1}+4\right) e_{\epsilon_{3}-\epsilon_{2}} e_{-\epsilon_{3}-\epsilon_{2}} e_{\epsilon_{2}-\epsilon_{1}} e_{\epsilon_{2}-\delta} \\
& -\left[\left(b_{1}-b_{2}\right)\left(b_{1}+b_{2}+3\right)+\left(b_{2}-b_{3}+2\right)\left(b_{2}+b_{3}+2\right)\right] e_{-\epsilon_{2}-\epsilon_{1}} e_{\epsilon_{2}-\epsilon_{1}} e_{\epsilon_{1}-\delta} \\
& -\left(b_{1}-b_{2}\right)\left(b_{1}+b_{2}+3\right) e_{\epsilon_{3}-\epsilon_{1}} e_{-\epsilon_{3}-\epsilon_{1}} e_{\epsilon_{1}-\delta} \\
& +\left(b_{1}+b_{2}+3\right)\left(b_{1}+b_{3}+2\right) e_{\epsilon_{3}-\epsilon_{2}} e_{\epsilon_{2}-\epsilon_{1}} e_{-\epsilon_{3}-\delta} \\
& +\left(b_{1}+b_{2}+3\right)\left(b_{1}-b_{3}+2\right) e_{-\epsilon_{3}-\epsilon_{2}} e_{\epsilon_{2}-\epsilon_{1}} e_{\epsilon_{3}-\delta} \\
& +\left[\left(b_{1}-b_{2}+1\right)\left(b_{2}+b_{3}+2\right)-\left(b_{1}-b_{2}\right)\left(b_{1}+b_{2}+3\right)\right] e_{\epsilon_{3}-\epsilon_{2}} e_{-\epsilon_{3}-\epsilon_{1}} e_{\epsilon_{2}-\delta}
\end{aligned}
$$




$$
\begin{aligned}
& +\left[\left(b_{1}-b_{2}+1\right)\left(b_{2}-b_{3}+2\right)-\left(b_{1}-b_{2}\right)\left(b_{1}+b_{2}+3\right)\right] e_{-\epsilon_{3}-\epsilon_{2}} e_{\epsilon_{3}-\epsilon_{1}} e_{\epsilon_{2}-\delta} \\
& +\left[\left(b_{1}-b_{2}\right)^{2}\left(b_{1}+b_{2}+3\right)+\left(b_{1}-b_{2}+1\right)\left(b_{2}-b_{3}+2\right)\left(b_{2}+b_{3}+2\right)\right] e_{-\epsilon_{2}-\epsilon_{1}} e_{\epsilon_{2}-\delta} \\
& +\left(b_{1}+b_{2}+3\right)\left(b_{1}-b_{3}+2\right)\left(b_{1}+b_{3}+2\right) e_{\epsilon_{2}-\epsilon_{1}} e_{-\epsilon_{2}-\delta} \\
& +\left(b_{1}-b_{2}\right)\left(b_{1}+b_{2}+3\right)\left(b_{1}+b_{3}+2\right) e_{\epsilon_{3}-\epsilon_{1}} e_{-\epsilon_{3}-\delta} \\
& +\left(b_{1}-b_{2}\right)\left(b_{1}+b_{2}+3\right)\left(b_{1}-b_{3}+2\right) e_{-\epsilon_{3}-\epsilon_{1}} e_{\epsilon_{3}-\delta} \\
& +\left(b_{1}-b_{2}+1\right)\left(b_{1}+b_{2}+3\right)\left(b_{1}-b_{3}+2\right)\left(b_{1}+b_{3}+2\right) e_{-\epsilon_{1}-\delta} .
\end{aligned}
$$

Then by a direct computation $S_{-\beta} v_{\lambda}^{+}$is the unique (up to a scalar multiple) singular vector in the Verma $\mathfrak{g}$-module $M(\lambda)$ of weight $\lambda-\beta$. It remains a challenging and interesting problem to find a closed formula for the singular vectors with respect to odd roots $\beta=\delta_{s}+\epsilon_{t}$ for general $\mathfrak{o s p ~ L i e ~ s u p e r a l g e b r a s . ~}$

\section{References}

1. S.-J. Cheng and W. Wang, Dualities and Representations of Lie superalgebras, Graduate Studies in Math., 144. American Mathematical Society, Providence, RI, 2012.

2. S.-J. Cheng and W. Wang, Character formulae in category $\mathcal{O}$ for exceptional Lie superalgebras $D(2 \mid 1 ; \zeta)$, Transform. Groups (2018). https://doi.org/10.1007/s00031018-9506-5, arXiv:1704.00846 4 .

3. J. Kujawa, Crystal structures arising from representations of $G L(m \mid n)$, Represent. Theory, 10 (2006), 49-85.

4. I. Musson, Šapovalov elements and the Jantzen sum formula for contragredient Lie superalgebras, arXiv:1710.10528.

5. T. Sale, Singular vector formulas for Verma modules of simple Lie superalgebras, $J$. Algebra, 521 (2019), 365-383.

6. V. Serganova, Kazhdan-Lusztig polynomials and character formula for the Lie superalgebra $\mathfrak{g l}(m \mid n)$, Selecta Math. (N.S.), 2 (1996), 607-651. 